MATEC Web of Conferences 25,03015 (2015)

DOI: $10.1051 /$ matec conf/ 20152503015

(C) Owned by the authors, published by EDP Sciences, 2015

\title{
Effect of Anode Structure on the Plasma Jet Characteristics of Arcjet Thruster
}

\author{
Zhenzhi Du, Xiong Chen, Yingkun Li \& Changsheng Zhou \\ School of Mechanical Engineering, Nanjing University of Science and Technology, Nanjing, Jiangsu, China
}

\begin{abstract}
In order to study the effects of anode geometry on volt-ampere characteristics and plasma jet characteristics of the arcjet thruster, the arcjet thruster flow characteristics were simulated. Through numerical simulation, the velocity field and temperature field of thruster were calculated, and the volt-ampere characteristics were obtained at different anode geometry. The calculation results show that the influence of the anode throat diameter was the most obvious, compared to the influence of the anode throat length and compression angle on volt-ampere characteristics and plasma jet characteristic of the arcjet thruster.
\end{abstract}

Keywords: arcjet thruster; anode structure; plasma jet; volt-ampere characteristics; numerical simulation

\section{INTRODUCTION}

In the arcjet thruster, gaseous propellant is heated by a DC electric arc to a temperature of about $1 \times 10^{4} \mathrm{~K}$, and the hot gas is subsequently expanded through a convergent-divergent nozzle, in which the gas's internal energy is converted into the kinetic energy of an axially-exhausting supersonic jet and thereby generates a thrust force. The arcjet thruster may achieve a specific impulse appreciably higher than that of the conventional chemical thruster ${ }^{[1,2]}$.

Over the past several decades, a lot of research has been devoted to the development of arcjet thruster and related technologies. Therefore, researchers accumulated a certain amount of experiment and numerical research work experience ${ }^{[3-8]}$. For instance, Yuan et al. ${ }^{[5]}$ carried out a numerical simulation of the DC arc plasma torch. Wang ${ }^{[6,7]}$ reported numerical simulation and experimental research data of low power arcjet thruster such as the internal flow and heat transfer characteristics. $\mathrm{Li}^{[8]}$ researched the influence of anode structure on arc plasma jet, but it is used in industrial cutting and welding, the structure of which is different from that of the arcjet thruster. In practical work, the anode structure is one of the important factors that affect arcjet thruster performance. However, there is no numerical study which is carried out about the influence of the anode structure on the plasma jet characteristics of the arcjet thruster.

In this paper, based on the magnetic vector potential of MHD model, we changed the anode throat diameter, throat length and anode contraction angle, and did numerical simulation on the characteristics of the plasma jet manifold and the volt ampere characteristics of the arcjet thruster. Through the numerical simulation, numerical results of the flow field in the arcjet thruster were acquired. The jet morphology and volt-ampere characteristics of different anode structure were compared, providing reference for the design and improving the anode structure of the arcjet thruster.

\section{PHYSICAL MODEL}

\subsection{Governing equation}

The main assumptions employed are as follows: (1) The gas flow in the arcjet nozzle is steady, axisymmetric, laminar and compressible; (2) the plasma is in the LTE state; (3) the plasma is optically thin; (4) the flow-induced electric field is negligible in comparison with the static electric field; (5) the azimuthal velocity component is negligible in comparison with the axial velocity component.

Based on these assumptions, the governing equations can be written as follows:

Mass equation is as follows:

$$
\frac{1}{r} \frac{\partial}{\partial r}(r \rho v)+\frac{\partial}{\partial x}(\rho u)=0
$$

Momentum equations are as follows:

$$
\begin{aligned}
& \frac{1}{r} \frac{\partial}{\partial r}(r \rho v u)+\frac{\partial}{\partial x}\left(\rho u^{2}\right)=-\frac{\partial P}{\partial x}+\frac{\partial}{\partial x}\left(2 \mu \frac{\partial u}{\partial x}\right) \\
& +\frac{1}{r} \frac{\partial}{\partial r}\left[r \mu\left(\frac{\partial u}{\partial r}+\frac{\partial v}{\partial x}\right)\right]+\left\{j_{r} B_{\theta}\right\} \\
& \frac{1}{r} \frac{\partial}{\partial r}\left(r \rho v^{2}\right)+\frac{\partial}{\partial x}(\rho v u)=-\frac{\partial P}{\partial r}+\frac{1}{r} \frac{\partial}{\partial r}\left(2 r \mu \frac{\partial v}{\partial r}\right)+ \\
& \frac{\partial}{\partial x}\left[\mu\left(\frac{\partial u}{\partial r}+\frac{\partial v}{\partial x}\right)\right]-2 \mu \frac{v}{r^{2}}-\left\{j_{x} B_{\theta}\right\}
\end{aligned}
$$

Energy equation is as follows: 


\section{MATEC Web of Conferences}

$\frac{\partial}{\partial r}\left(r \rho c_{p} v T\right)+\frac{\partial}{\partial x}\left(\rho c_{p} u T\right)=\frac{1}{r} \frac{\partial}{\partial r}\left(r k \frac{\partial T}{\partial r}\right)+\frac{\partial}{\partial x}\left(k \frac{\partial T}{\partial x}\right)$

$+\left\{\frac{5 k_{B}}{2 e}\left(j_{r} \frac{\partial T}{\partial r}+j_{x} \frac{\partial T}{\partial x}\right)+\frac{j_{r}^{2}+j_{x}^{2}}{\sigma}-4 \pi \varepsilon_{N}\right\}$

Where: $x$ and $r$ are the axial and radial coordinate; $\rho$ is density; $u$ and $v$ are the axial and radial velocity component; $\mu$ is viscosity coefficient, $T$ and $P$ are respectively the temperature and the pressure. In the energy equation, $k, c_{p}, \sigma, \varepsilon_{N}, k_{B}$ and $e$ are respectively the thermal conductivity, specific heat at constant pressure, electrical conductivity, net radiation coefficient, Boltzmann constant and elementary charge.

Electric potential (current continuity) equations are as follows:

$\frac{1}{r} \frac{\partial}{\partial r}\left(r \sigma \frac{\partial \varphi}{\partial r}\right)+\frac{\partial}{\partial x}\left(\sigma \frac{\partial \varphi}{\partial x}\right)=0$

$j_{x}=-\sigma \frac{\partial \varphi}{\partial x}, j_{r}=-\sigma \frac{\partial \varphi}{\partial r}$

Magnetic vector potential equations are as follows:

$\frac{1}{r} \frac{\partial}{\partial r}\left(r \frac{\partial A_{x}}{\partial r}\right)+\frac{\partial}{\partial x}\left(\frac{\partial A_{x}}{\partial x}\right)=-\mu_{0} j_{x}$

$\frac{1}{r} \frac{\partial}{\partial r}\left(r \frac{\partial A_{r}}{\partial r}\right)+\frac{\partial}{\partial x}\left(\frac{\partial A_{r}}{\partial x}\right)=-\mu_{0} j_{r}+\frac{A_{r}}{r^{2}}$

Where: $\mu_{0}$ is the permeability of free space; $\varphi$ is the electric potential; $j_{x}$ and $j_{r}$ are the axial and radial electric current density; $A_{x}$ and $A_{r}$ are respectively the axial and radial magnetic vector potential.

The special source term about plasma transport is added in the momentum and energy equations. Special source term in the momentum equations are axial and radial Lorentz force. Special source term in energy equation includes the electronic enthalpy transport items, Joule heat and Radiation loss. The above equations constitute the closed equations of the MHD (Magnetohydrodynamics) model.

\subsection{Boundary conditions}

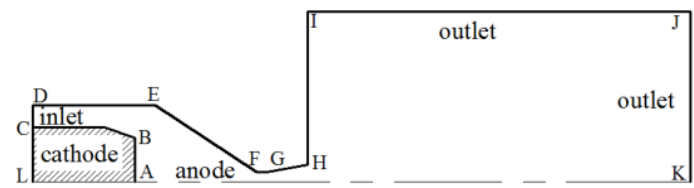

Figure 1. Schematic diagram of the arcjet thruster

Figure 1 is a schematic diagram of the arcjet thruster under study. The nozzle is easy to machining, and it can obtain the supersonic plasma jet.

Using the terms of the MHD model based on the magnetic vector potential, the boundary conditions are shown in Table 1. Vector potential boundary conditions aren't quite strict in math, but they aren't against Ampere's law, so they are widely used in the numerical simulation of plasma $[9,10]$. The working medium adopted by the arcjet thruster is nitrogen and the thermodynamic and properties of nitrogen plasma are obtained from the literature ${ }^{[11]}$. Cathode current density in the table is given according to the K. C. Hsu calculation method ${ }^{[12]}$, and $j_{\text {given }}$ is set to $2.64 \times 10^{8} \mathrm{~A} / \mathrm{m}^{2}(150 \mathrm{~A})$.

\section{RESULTS AND DISCUSSION}

In this paper, the influence of the anode structure parameters on the volt ampere characteristic and plasma jet of the arcjet thruster is studied. The anode structure parameters include throat diameter $d$, throat length $l$ and the anode contraction angle $\alpha$.

Table 1. MHD model boundary conditions

\begin{tabular}{|c|c|c|c|c|c|c|}
\hline & $\mathbf{U}$ & $P$ & $T$ & $\varphi$ & $A_{x}$ & $A_{r}$ \\
\hline $\mathbf{A B}$ & 0 & - & $3000 \mathrm{~K}$ & $j=j_{\text {given }}$ & $\frac{\partial A_{x}}{\partial r}=0$ & $\frac{\partial A_{r}}{\partial r}=0$ \\
\hline CD & $\begin{array}{l}\text { Mass flow } \\
7.1 \mathrm{~g} / \mathrm{s}\end{array}$ & $5.0 \times 10^{5} \mathrm{~Pa}$ & $300 \mathrm{~K}$ & $\frac{\partial \varphi}{\partial r}=0$ & $\frac{\partial A_{x}}{\partial r}=0$ & $\frac{\partial A_{r}}{\partial r}=0$ \\
\hline FG & 0 & - & $1000 \mathrm{~K}$ & $\varphi=0$ & $\frac{\partial A_{x}}{\partial r}=0$ & $\frac{\partial A_{r}}{\partial r}=0$ \\
\hline IJK & - & $1.0 \times 10^{5} \mathrm{~Pa}$ & $300 \mathrm{~K}$ & $\frac{\partial \varphi}{\partial r}=0$ & 0 & 0 \\
\hline $\mathbf{A K}$ & $\frac{\partial u}{\partial r}=0, v=0$ & - & $\frac{\partial T}{\partial r}=0$ & $\frac{\partial \varphi}{\partial r}=0$ & $\frac{\partial A_{x}}{\partial r}=0$ & $\frac{\partial A_{r}}{\partial r}=0$ \\
\hline
\end{tabular}




\subsection{Effect of anode throat diameter d on thruster}

The volt-ampere characteristics, the temperature and velocity distribution on the arcjet thruster axis are shown in Figures 2-4. When the anode contraction angle is $60^{\circ}$, the throat length $l$ is $1.0 \mathrm{~mm}$, and the throat diameter $d$ is respectively $2.0 \mathrm{~mm}, 2.5 \mathrm{~mm}$, $3.0 \mathrm{~mm}$ and $4.0 \mathrm{~mm}$.

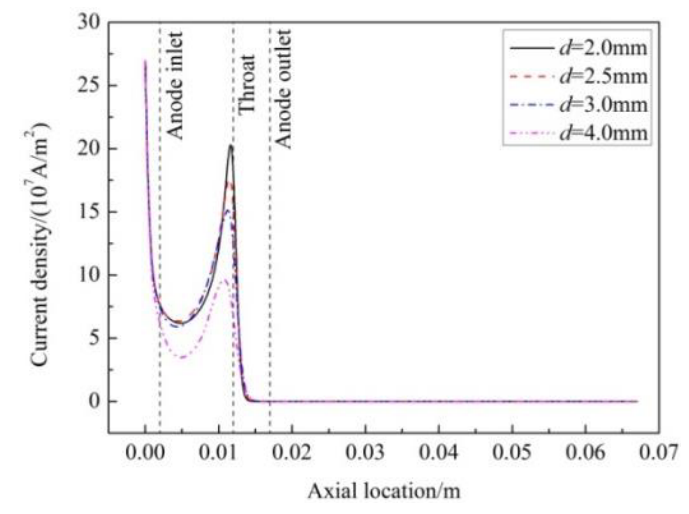

(a) Distribution of current density along the arcjet thruster axis

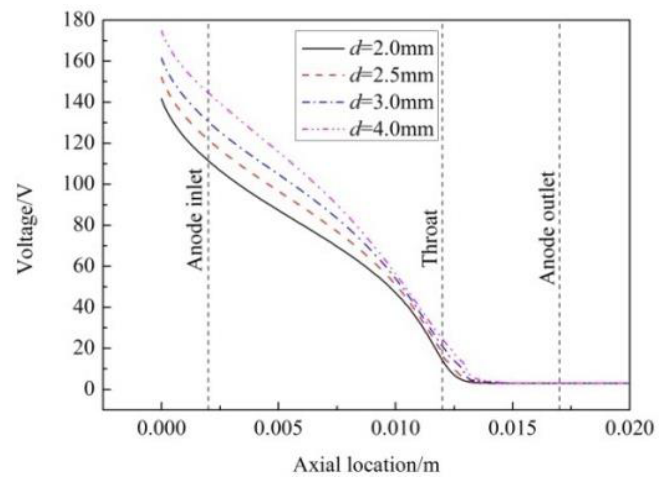

(b) Distribution of voltage along the arcjet thruster axis

Figure 2. Comparison of volt-ampere characteristics of the arcjet thruster

As shown in Figure 2, the diameter of the anode throat has a great influence on the volt-ampere characteristics of the arcjet thruster. Arc voltage of arcjet thruster increases with the increasing anode throat diameter. This is because with the increase of throat diameter, the volume which is near the throat increases and the current density decreases, weakening the Joule heating effect. And the arc temperature decreases, decreasing the conductivity of nitrogen, thus the impedance increases, eventually leading to arc voltage increase.

From Figure 3, it can be seen that the temperature distribution on the arcjet thruster axis of different throat diameters is different and the temperature of the arcjet thruster decreases obviously with the increase of throat diameter. When the diameter is $2.0 \mathrm{~mm}$, the stronger mechanical compression effect and the higher current density result in strong Joule heat effect. So when the maximum temperature on the arcjet thruster axis reaches $17643 \mathrm{~K}$ and the diameter is $4.0 \mathrm{~mm}$, the maximum temperature is dropped to $11732 \mathrm{~K}$. Namely, in the case of other dimensions remain unchanged, mechanical compression effect and Joule heating effect of nozzle are enhanced with throat diameter decreasing, resulting in plasma in the anode nozzle temperature increases.

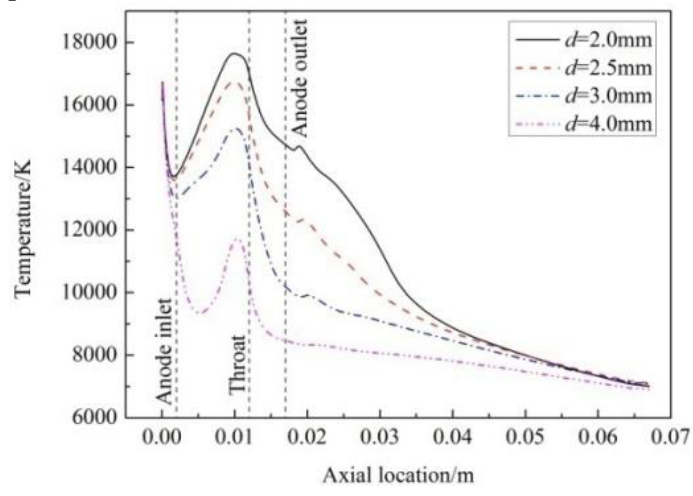

Figure 3. Distribution of temperature along the arcjet thruster axis

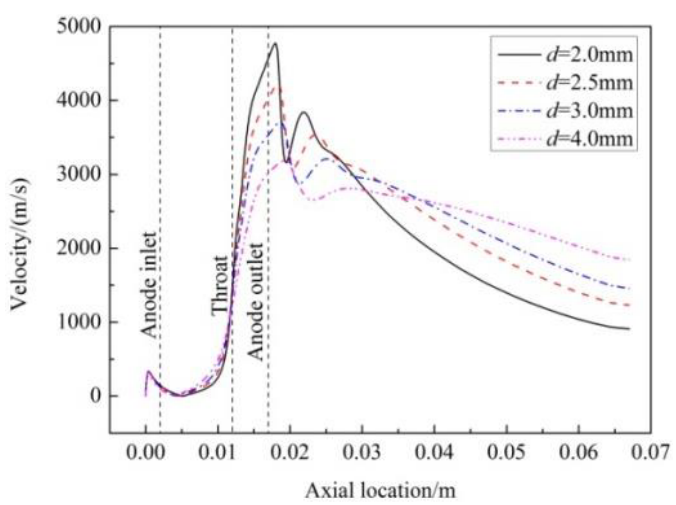

Figure 4. Distribution of velocity along the arcjet thruster axis

As shown in Figure 4, the plasma velocity at the arcjet thruster axis from the anode throat position appears more differences. Since the size of the throat diameter impact the thruster temperature field, different throat diameter has a certain influence on the thruster velocity field. As described above, when the nozzle throat diameter decreases, the nozzle of mechanical compression effect and Joule heating effect are enhanced and the temperature of the thruster increases, the density of the plasma is reduced by the law of conservation of mass and energy conservation, and the plasma velocity correspondingly increases. When the throat diameter is $2.0 \mathrm{~mm}$, the maximum 


\section{MATEC Web of Conferences}

speed is $4774 \mathrm{~m} / \mathrm{s}(\mathrm{Ma}=2.27)$. When the throat diameter is $4.0 \mathrm{~mm}$, the maximum speed is $3187 \mathrm{~m} / \mathrm{s}$ $(\mathrm{Ma}=2.00)$. As can be seen from the graph, the smaller the throat diameter is, the larger the maximum speed is, and the stronger the shock wave can be.

\subsection{Effect of anode throat length l on thruster}

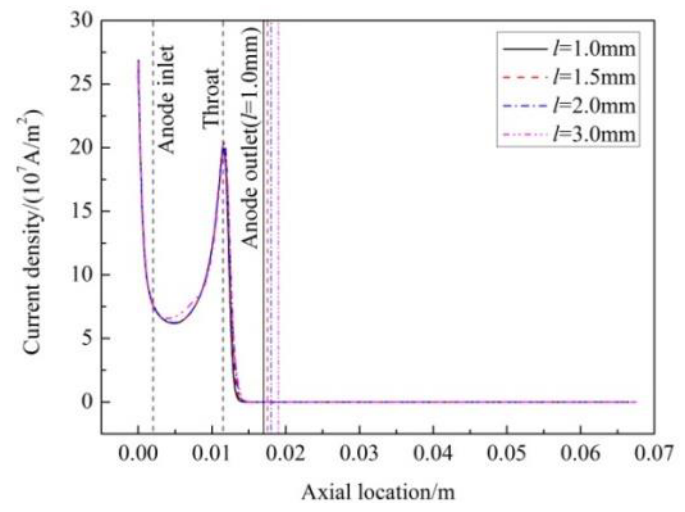

(a) Distribution of current density along the arcjet thruster axis

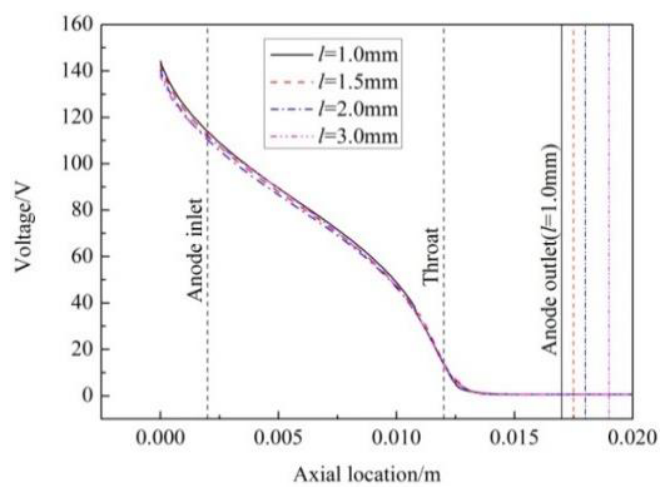

(b) Distribution of voltage along the arcjet thruster axis

Figure 5. Comparison of volt-ampere characteristics of the arcjet thruster

The volt-ampere characteristics, the temperature and velocity distribution on the arcjet thruster axis are shown in Figures 5-7. When the anode contraction angle is $60^{\circ}$, the throat diameter $d$ is $2.0 \mathrm{~mm}$, and the throat length $l$ is respectively $1.0 \mathrm{~mm}, 1.5 \mathrm{~mm}, 2.0 \mathrm{~mm}$ and $3.0 \mathrm{~mm}$.

In Figure 8, the anode contraction angle and throat diameter of arcjet thruster remain unchanged, changing the throat length which has little effect on the volt-ampere characteristics of the arcjet thruster. The current density distribution and arc voltage of different throat lengths are basically consistent; the arc voltage is respectively $142 \mathrm{~V}(1.0 \mathrm{~mm}), 141 \mathrm{~V}(1.5 \mathrm{~mm}), 140 \mathrm{~V}$ $(2.0 \mathrm{~mm})$ and $138 \mathrm{~V}(3.0 \mathrm{~mm})$. Compared with throat diameter, the influence of throat length on the volt ampere characteristics of the thruster can be neglected.

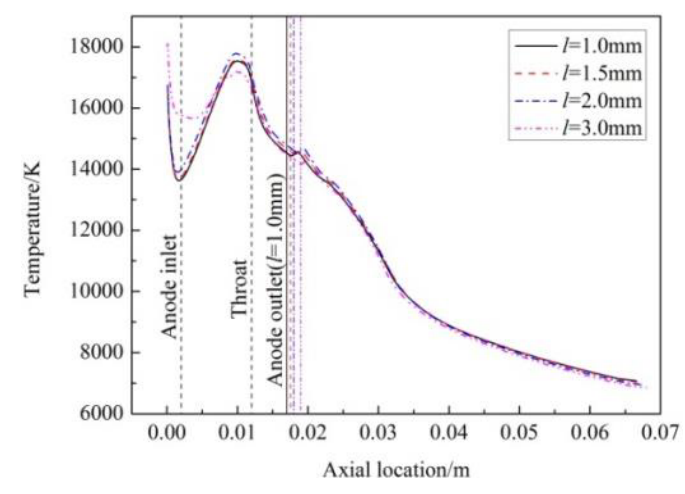

Figure 6. Distribution of temperature along the arcjet thruster axis

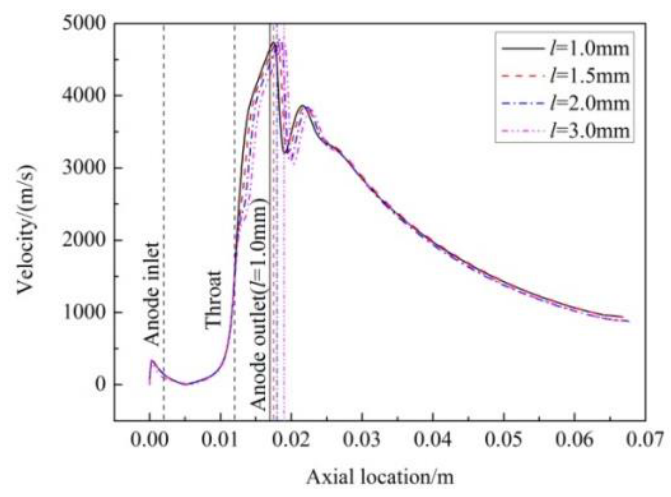

Figure 7. Distribution of velocity along the arcjet thruster axis

From Figure 6, it can be found that when the throat length is respectively $1.0 \mathrm{~mm}, 1.5 \mathrm{~mm}$ and $2.0 \mathrm{~mm}$, the difference of the temperature distribution on the arcjet thruster axis is small; with the increase of throat length, the temperature on the arcjet thruster axis increases slightly. This is because with the increasing throat length, the compression effect of the nozzle leads to a temperature increase, but the temperature of the engine is mainly determined in Joule heat, so the temperature increase is small. When the throat length is $3.0 \mathrm{~mm}$, the temperature distribution on the arcjet thruster axis is different than before. In the vicinity of the cathode surface and the anode inlet, the temperature is significantly higher than that of the other three throat lengths, while the temperature at the entrance of the throat is less than that of the other three species. The heat energy absorbed by the wall of the anode increases with the increase of the throat length, which leads to a decrease in temperature.

From Figure 7, it can be seen that the change of the throat length has little effect on the plasma velocity distribution along the arcjet thruster axis. As is mentioned above, changing the throat length has little effect on the current density and temperature field of 
arcjet thruster, so the impact on the thruster velocity field is smaller.

\subsection{Effect of anode contraction angle $\alpha$ on thruster}

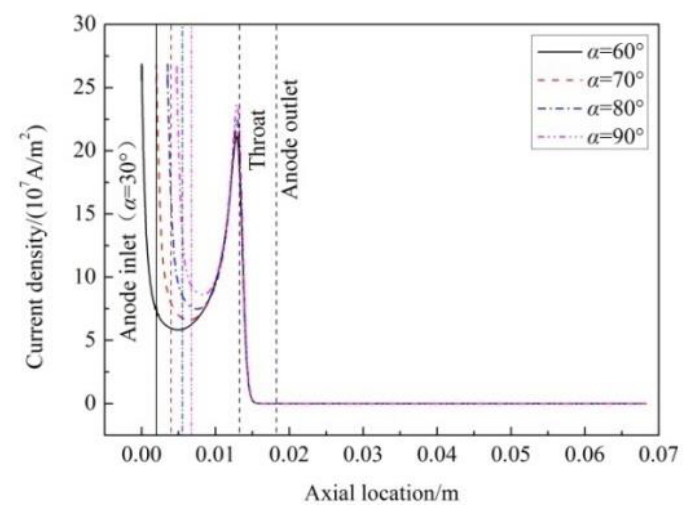

(a) Distribution of current density along the arcjet thruster axis

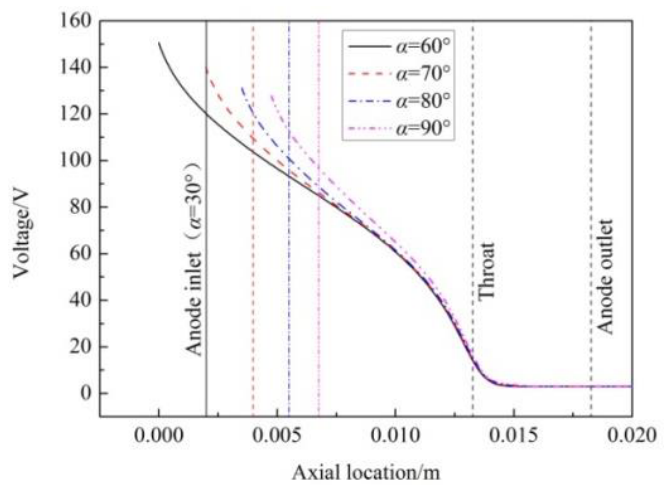

(b) Distribution of voltage along the arcjet thruster axis

Figure 8. Comparison of volt-ampere characteristics of the arcjet thruster

The throat diameter $d$ is $2.0 \mathrm{~mm}$; the throat length $l$ is $1.0 \mathrm{~mm}$; the anode contraction angle $\boldsymbol{\alpha}$ is respectively $60^{\circ}, 70^{\circ}, 80^{\circ}$ and $90^{\circ}$. The volt-ampere characteristics, the temperature and velocity distribution on the arcjet thruster axis are shown in Figures 8-10.

From Figure 8, the anode contraction angle has great influence on the volt-ampere characteristics of the arcjet thruster. In particular, the influence on arc voltage is great, which decreases with the increase of the anode contraction angle. When the inlet diameter and throat diameter of the anode remain unchanged, changing the angle of the anode angle, it means that the length of the contraction section is actually changed. Therefore, the distance between cathode and anode is changed. So as the anode contraction angle becomes smaller, the length of the contraction section increases and the distance between cathode and anode increases. This eventually led to an increase in engine power and the arc voltage increase at a constant working current situation

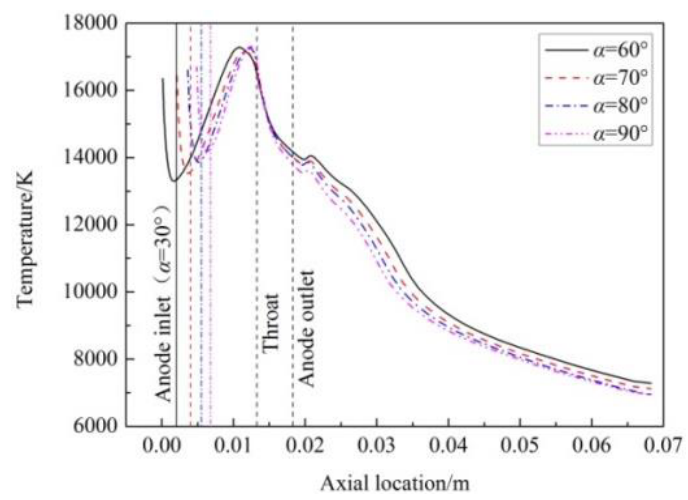

Figure 9. Distribution of temperature along the arcjet thruster axis

It can be seen from Figure 9 that the anode contraction angle has little effect on the temperature distribution of the arcjet thruster. Since the temperature field of arcjet thruster mainly depends on the current density distribution, the anode contraction angle has little effect on the current density distribution of the arcjet thruster, so the temperature distribution inside thruster is less affected. Anode contraction angle has some effect on the temperature distribution of the thruster outside: The smaller angle of anode contraction section is, the higher temperature of the external arcjet thruster will be. That is because the smaller the anode converging angle is, the stronger the compression effect of plasma is, and the smaller the flame radius is, then energy is mainly concentrated in the vicinity of the central axis.

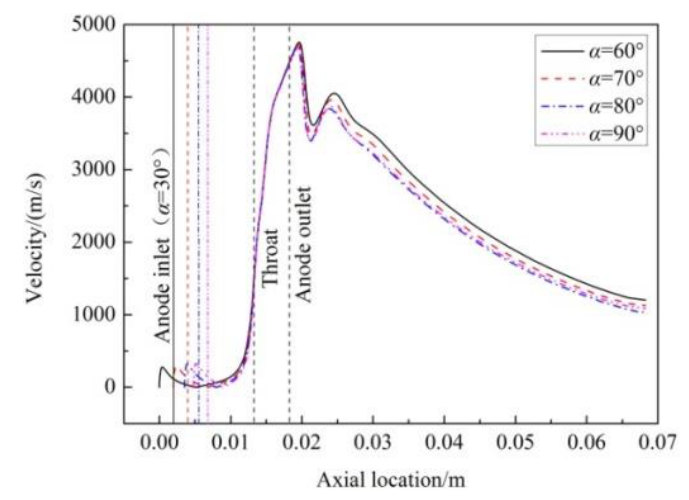

Figure 10. Distribution of velocity along the arcjet thruster axis

As described above, the change of the anode contraction angle only has an effect on the temperature distribution of the external flow field. The influence of the temperature distribution of the inner flow field can be neglected, and the thruster velocity distribution is mainly determined by the temperature field. It is 


\section{MATEC Web of Conferences}

shown from Figure 10 that the influence of the changing anode contraction angle on the velocity distribution of the arcjet thruster is consistent with the temperature distribution.

\section{CONCLUSIONS}

A numerical research was carried out to compare the plasma jet and volt-ampere characteristics of different anode structure parameters. Through this study, we obtain the following conclusions. With the increase of throat diameter for the same anode contraction angle and throat length, the current density on the arcjet thruster axis is reduced, the arc voltage increases, and the highest temperature and the maximum velocity of the plasma on the arcjet thruster axis are decreased. Moreover, the influence of throat diameter on the volt-ampere characteristics and plasma jet characteristics of the arcjet thruster is very obvious. However, the influence of throat length and anode contraction angle on the volt-ampere characteristics and plasma jet characteristics of the arcjet thruster is negligible.

\section{REFERENCES}

[1] Sackheim R L, \& Sackheim R L. 2006. Overview of united states space propulsion technology and associated space transportation systems. Journal of Propulsion and Power, 22(6):1310-1333.

[2] Butler G W, \& Cassady R J. 1996. Directions for arcjet technology development. Journal of Propulsion and Power, 12(06): $1026-1034$
[3] Horisawa H, \& Kimura I. 2000. Very low-power arcjet testing. Vacuum, 59(1): 106-117.

[4] Pobst J A, Wysong I J, \& Spores R A. 1995. Laser induced fluorescence of ground state hydrogen atoms at nozzle exit of an arcjet thruster. AIAA Paper, 95-1973.

[5] Yuan Xingqiu, Li Hui, \& Zhao Taizhe, et al. 2004. Numerical study of supersonic plasma torch. Journal of Physics, 53(3): 788-792.

[6] Wang Haixing, Chen Xi, \& Pan Wenxia, et al. 2010. Modeling study on the flow, heat transfer and energy conversion characteristics of low-power arc-heated hydrogen/ nitrogen thrusters. Plasma Chemistry and Plasma Processing, 30(6): 707-731.

[7] Wang Haixing, Wei Fuzhi, \& Murphy A B, et al. 2012. Numerical investigation of the plasma flow through the constrictor of arc-heated thrusters. Journal of Physics D. Applied Physics, 45(23): 6705-6711.

[8] Li Deyuan, Han Hailing, \& DONG Xiaoqiang. 2010. Influence of structure and dimension of plasma gun nozzle on arc energy. Journal of Shenyang University of Technology, (2):126-130.

[9] Lago F, Gonzalez J J, \& Freton P, et al. 2004. A numerical modelling of an electric arc and its interaction with the anode: part I. the two-dimensional model. Journal of Physics D: Applied Physics, 37(6): 883-897.

[10]Bini R, Monno M, Boulos M I. Numerical and experimental study of transferred arcs in argon. Journal of Physics D: Applied Physics, 3253, 39(15): 3253-3266.

[11]Wang Weizong, Rong Mingzhe, \& B.Murphy A, et al 2010. Computation Analysis on Properties of High Temperature Nitrogen Arc Plasmas. High Voltage Engineering, 36(11): 2777-2784

[12]K.C.Hsu, K.Etemadi, \& E.Pfender. 1983. Study of the free burning high intensity argon arc. Applied Physics, 54, 1293-1301. 